the use of American trade names for drugs means that one example outlined is not understandable to a wider readership. Those with some knowledge of the field will find some points of interest in $\mathrm{Dr}$ Dyer's exploration of the underlying theme. It is not a suitable book on its own as an introduction to ethics for trainee psychiatrists.

The book is published only in hardback at $£ 35$ and is worthwhile for a library purchase.

J M PARROTT Consultant Forensic Psychiatrist, Bexley Hospital

\section{Biomedicine Examined}

\author{
Edited by Margaret Lock and \\ Deborah Gordon, 558 pages, \\ Dordrecht, £56.00, hbk, Kluwer \\ Academic Publishers, 1988
}

In the last decade biomedicine has attracted a good deal of critical attention, not least because of its cost implications. Commentators have become reluctant to accept on trust claims made in its name. This is partly a function of the growing realisation that biomedical knowledge and practices are social accomplishments, rather than 'natural' or 'given' phenomena, and as such open to analysis and critique. As one of the editors of Biomedicine Examined, Deborah Gordon, puts it: 'We may increasingly speak of a social scientific/historic gaze turned on medicine, describing hidden cultural scaffolding and social processes that shape practice and knowledge' (page 20).

In this volume the editors have put together a collection of papers from anthropologists, sociologists and physicians on the interdependence of jiomedicine, society and culture. More specifically, the emphasis is on a largely ethnographic approach to 'the meanings and values implicit in biomedical knowledge and practice'. Margaret Lock and Deborah Gordon have done their job admirably, and the eighteen varied and challenging papers assembled will constitute a rich resource for health academics and practitioners.

The papers cover aspects of the reproduction of medical knowledge, changes and recent extensions in the jurisdiction of physicians, the 'routinisation' of medical technology, the relevance of social and cultural context to biomedical practice, and modes of legitimation of biomedicine. They are of a uniformly high standard and there should be something in this volume for everyone with a critical interest in issues of health and health care. Perhaps one theme, which recurs in a number of papers, will be of particular interest to readers of this journal. This is the notion that the consolidation of biomedicine in Western health care has led to a situation in which its practitioners, claiming expertise through science, have come to proffer scientific solutions to problems which are essentially moral or political, and hence the province of us all.

It would be invidious to pick out papers for special mention here for two reasons: first, the overall quality of this 'large' and heterogeneous collection is high; and second, what individual readers find useful and helpful will be contingent on their own theoretical and practical interests. I have no hesitation, however, in recommending this impressive text to all those with a genuine concern for the future of 'healing'.

\section{GRAHAM SCAMBLER, Senior Lecturer in Sociology Academic Department of Psychiatry University College and Middlesex School of Medicine, London.}

\section{The Genetics of Mental Retardation}

\section{Edited by $\mathrm{E}$ K Hicks and J M Berg, 207 pages, Dordrecht, $\$ 69.00$, hbk, Kluwer Academic Publishers, 1988}

This book comprises the papers and brief discussion summaries of an international workshop which convened in 1986. The title is somewhat misleading. The book is divided into three sections: Prenatal Diagnosis, Postnatal diagnosis and Genetic counselling. Each section is a combination of purely scientific or medical accounts, such as the chromosomal findings in first trimester chorionic villus biopsy, and the use of the computer in the diagnosis of dysmorphic syndromes, and papers on moral and psychosocial aspects: for example, ethical questions in the prenatal chromosomal diagnosis of mental retardation and psychosocial developments in the field of genetic counselling. Each paper is of high quality, as would be expected from the distinguished body of contributors. There are particular gems from $\mathbf{R}$ W Smithells, reflecting sensitively on the management of both child and parents when handicap is diagnosed immediately after birth, and from $G R$ Dunstan on ethical issues in the field of choice in treating the severely handicapped newborn child, drawing out that the final arbiter in management is the physician as the 'authorised moral agent'. J C Fletcher explores how ethical issues evolve in differen societies in the practice of prenatal diagnosis, using data derived from his multi-nation, multi-culture survey of medical geneticists.

Prenatal diagnosis and genetic $\vec{\sigma}$ counselling have long been topics of i ethical discussion, and much has been written on the subject. This was why it was a disappointment in a way to open a book entitled The Genetics of Mental Retardation and find it was, in fact, this well-worked field. Further, Genetics is a rapidly advancing subject, and today in vitro fertilisation and genetic manipulation of embyros have superseded, although not eliminated prenatal diagnosis and genetio counselling as prime areas of morat concern at the beginning of life, whiff still leading to discussions on the sanctity and quality of life. Yet they are scarcely mentioned. Nevertheless, this is a useful and authoritative collection of papers which are a pleasure to read.

\section{Reader in Develpmental Genetics Division of Medical and Molecular Genetics Guy's Hospital}

\section{Manuale di Bioetica (2nd Edition)}

\author{
Elio Sgreccia, 542 pages, Milan, \\ L 44,000, Vita e Pensiero, 1988 \\ (Published in Italian)
}

Bioethics as a discipline has become more and more topical and interesting in the last few years, because of new problems, and today it is at a significant turning point. It has now passed from the universities to political and parliamentary sessions, although actually this is more a return to the political environment. We know, in fact, that bioethics was born partly as a result of the Nurenberg trial, which brought to light the terrible crimes of 
the Nazi doctors. The purpose of bioethics is to give theoretical weight to the proclamation of some of man's basic rights which began to be formulated after the 'universal declaration of the rights of man' in 1948, the 40th anniversary of which was recently celebrated.

And thus bioethics aims to study 'human conduct in the area of the life sciences and health care, insofar as this conduct is examined in the light of moral values and principles'.

This handbook of bioethics, by Professor Elio Sgreccia, the director of the first Italian University Centre for Boethics, fits into that perspective. The handbook appears at a distance of two years from the first edition and it constitutes a revision and update of the first edition.

As the author explains in the preface, a re-elaboration of the previous volume has been necessary, because in the last few years important documents have been issued concerning problems of bioethics that could not be ignored. At the end of 1986, the Parliamentary Assembly of the Council of Europe issued Recommendation number 1046 which touched on the delicate problem of the experimentation and use of human embryos in the biomedical field. In January 1987 the International Conference of the European Medical Associations approved the almost statutory document, 'European Principles of Medical Ethics' which deals with all the main points of bioethics. In the Catholic field, the Congregation for the Doctrine of the Faith has published the 'Instruction on respect for human life in its origin and on the dignity of procreation', a document which expresses the position of the Catholic Church on the question of reproductive techniques and in the area of the very basis of bioethics (for example the clear definition of the human embryo's identity).

The first part of the handbook (chapters I-V), deals with the foundations and principles of bioethics, the problems of the origin of life, the centrality of the human being and the value of his physical existence and the position of bioethics within the complexity of biomedicine. In the detailed part (chapters VI-XV) the main themes of bioethics - genetic engineering, reproductive techniques, experimentation on man and on embryos, organ transplants, transsexualism, euthanasia etc, are widely discussed.

At the end of every chapter there is a broad and detailed bibliography; the subject index and the author's index make the volume very useful for rapid consultation.

The worthy aim of the handbook is to offer methodological help to all who approach bioethics and its problems or who want a deeper knowledge of the subject. The readers will not only find, however, a panorama of the various positions on individual problems (which many other manuals of this kind often limit themselves to): they will also find the author's position clearly exposed, without ambiguity. He considers his position valid because of the reasoning supporting it; reasoning which, without mystery or feigning, recalls the personalistic philosophy, faithful both to science and to philosophical reasoning, founded on the anthropology of the totality of man.

In this philosophical tradition the author saw that it was legitimate to refer constantly to the documents of the Magisterium of the Catholic Church, both because the Catholic Church's view of faith does not disturb autonomous rational reflection and because the Catholic Church's Magisterium itself has actually brought a wide and deep contribution to bioethics. Also the author felt that not to quote at least the most interesting positions of the Catholic Church and the most well known documents would have been to display a lack of informative objectivity.

Finally, what one will be able to see in every page of the manual, is the author's conviction that there cannot and must not be a conflict between scientific truth and ethical truth, so that the 'bridge to the future', which bioethics represents, can certainly lead to a new humanism, bringing into harmony the values of science and of technology and those of man's creativity and freedom as well as those of respect for his dignity and substantial integrity.

DR ANTONIO G SPAGNOLO Fellow, Centre for Bioethics, Catholic University, 1, L Francesco Vito, 00168 Rome, Italy.

\section{Nurses, Gender and Sexuality}

Jan Savage, 168 pages, London, £8.95, Heinemann Nursing, 1987.

This book explores the nature of nursing as applied to all aspects of sexuality, encompassing the gender and sexuality of both nurses and patients.
The author maintains that the issue of sexuality, often brushed aside by nurse educationalists in the past, and thus nurses themselves, forms an integral part of the nurse - patient relationship. Aspects fundamental to care are explored constuctively.

In the introduction, the author describes the motivating forces which led her to write this book. She wrote it primarily because she lacked information which she might otherwise have used in order to help patients following gynaecological surgery, and secondly in order to reduce the uneasiness that many nurses feel concerning this sensitive issue.

Chapter Two gives an in-depth explanation of the nature of sexuality, using arguments from disciplines allied to nursing, such as social anthropology, sociology, psychology and philosophy.

The book proceeds by discussing the concepts of erotic and non-erotic sexuality in health and illness. Related topics such as body image and selfconcept are explored, followed by a description of the effects of sickness on the individual's capacity for sexual expression. Most usefully, the author includes specific suggestions as to the ways by which the sick person might still fulfil his/her sexual potential.

The author continues by tracing the history of the roles assigned to both male and female members of the nursing profession, together with the resulting sexual stereotyping which may be observed today. The author suggests that 'female' should no longer be synonymous with 'handmaiden' within a nursing sphere, and points to the confusion felt by many male doctors and patients when confronted by male nurses. The issue of homosexuality, both male and female, is raised, and the ethical aspects of this matter are highlighted; there are disturbing and thought-provoking accounts of situations where nursing care had been inadequate because of the patient's 'deviation' from the 'normal' sexual role.

The author proceeds by questioning the right of nursing and medical staff to ignore patients' need for privacy, not only in an acute hospital setting, but also in long-term institutional care, thus denying their possible wishes for any degree of intimacy with their partner.

Towards the end of the book, the emphasis shifts in order to include a presentation of a care study, focussing on expressing sexuality and utilising an appropriate nursing model in order to meet the needs of the hypothetical patient discussed. 